\title{
Carminella Biondi, Elena Pessini, Rêver le monde, écrire le monde. Théorie et narrations d'Édouard Glissant
}

\section{Francesca Torchi}

\section{(2) OpenEdition \\ 12 Journals}

\section{Édition électronique}

URL : http://journals.openedition.org/studifrancesi/36826

DOI : $10.4000 /$ studifrancesi.36826

ISSN : 2421-5856

Éditeur

Rosenberg \& Sellier

\section{Édition imprimée}

Date de publication : 1 juillet 2005

Pagination : 209-210

ISSN : 0039-2944

\section{Référence électronique}

Francesca Torchi, «Carminella Biondi, Elena Pessini, Rêver le monde, écrire le monde. Théorie et narrations d'Édouard Glissant », Studi Francesi [En ligne], 145 (XLIX | I) | 2005, mis en ligne le 30 novembre 2015, consulté le 18 avril 2021. URL : http://journals.openedition.org/studifrancesi/36826 : DOI : https://doi.org/10.4000/studifrancesi.36826

Ce document a été généré automatiquement le 18 avril 2021.

\section{cc) $(9)$}

Studi Francesi è distribuita con Licenza Creative Commons Attribuzione - Non commerciale - Non opere derivate 4.0 Internazionale. 


\section{Carminella Biondi, Elena Pessini, Rêver le monde, écrire le monde. Théorie et narrations d'Édouard Glissant}

Francesca Torchi

\section{RÉFÉRENCE}

CARMINELLA BIONDI, ELENA PESSINI, Rêver le monde, écrire le monde. Théorie et narrations d'Édouard Glissant, Bologna, Clueb, 2004, 146 p.

1 L'année 2004 s'impose comme une date importante pour les rapports d'Édouard Glissant et de son œuvre avec le lectorat italien. Avec un certain retard par rapport à d'autres pays, le rôle fondamental de cette production littéraire dans le panorama de la littérature contemporaine commence à y être reconnu. Les traducteurs s'essayent enfin à appréhender les romans et les essais théoriques de l'écrivain martiniquais, et les résultats ne se font pas attendre. Le Quatrième siècle remporte le prix Grinzane Cavour, et l'université de Bologne attribue à l'auteur la Laurea ad Honorem au cours d'une cérémonie qui a eu lieu au mois de juin 2004. Ce succès, tardif il est vrai, s'explique par le fait que la pensée glissantienne, qui s'est élaborée et forgée au cours d'une cinquantaine d'années, propose une réflexion qui embrasse les problématiques et les défis que vit le monde dans sa globalité; elle s'offre comme guide et comme éclaireur à un lectorat désireux de décrypter les enjeux que posent les nouvelles relations entre les hommes, les espaces et les temps. Le volume de Carminella Biondi et Elena Pessini réunit des essais qui avaient déjà été publiés précédemment (sauf un essai inédit d'Elena Pessini), mais qui sont ici revus et mis à jour au sein d'un ensemble organisé en trois grandes parties: "Théoriser", "Raconter les Antilles", "Raconter le monde". Dans la première section dialoguent et se répondent deux articles, respectivement de chacun des auteurs, qui interrogent les grandes idées glissantiennes s'exprimant dans les écrits 
théoriques de l'écrivain. Une théorie dont les critiques soulignent le caractère de système "ouvert" et dynamique, refusant les schémas préétablis, en évolution continuelle. C'est entre autres le concept de "créolisation" qui est examiné, depuis sa naissance dans les textes jusqu'à ses dernières acceptions. En effet, ce concept charpente la réflexion glissantienne et s'enrichit de multiples facettes; il témoigne de la complexité du monde mais ce principe est également à la base de l'écriture et de la recherche stylistique de Glissant qui tentent justement de rendre compte de cette complexité. Les nombreux essais théoriques d'Édouard Glissant, depuis Soleil de la conscience (1956) jusqu'au Traité du Tout-monde (1997), élaborent une vision qui permet de "lire non seulement l'histoire du peuple antillais mais aussi (de) lire les changements du monde, les comprendre, les prévoir, les vivre" (p. 13). Selon Glissant, l'écrivain doit s'engager à "explorer l'inconnu, le non-familier, le contraire de soi" (p. 16), pratiquer l'errance, tant mentale que spatiale, pour donner forme à la Relation, cette nouvelle hypothèse de rencontre avec l'autre, "sans qu'il y ait perte d'identité, mais, au contraire, acquisition d'une identité nouvelle et plus riche, douée d'une capacité d'enracinement multiple dans différents espaces géographiques, culturels et linguistiques" (p. 28).

2 Après avoir parcouru la pensée d'Édouard Glissant présente dans les essais, les auteurs proposent, dans les deux sections qui suivent, une analyse de l'œuvre romanesque, avec des incursions dans d'autres genres, en particulier dans le grand poème Les Indes (1956). Les titres de ces deux parties tracent un parcours idéal qui nous conduit des narrations qui se concentrent essentiellement sur les Antilles, et la Martinique en particulier, à un contexte de plus en plus vaste. Le corpus de la deuxième partie "Raconter les Antilles" est constitué par les récits qui mettent en scène la condition de la Martinique et de son peuple, qui s'interrogent sur l'Histoire et l'identité de ce territoire. Carminella Biondi souligne que c'est dans Les Indes que germe l'idée des Antilles comme "laboratoire du monde à venir. Glissant voit en effet dans les Antilles, où tant d'ethnies et de cultures s'affrontent et se confrontent, un creuset où il serait possible d'expérimenter des transmutations fécondes à partir des données culturelles souvent contradictoires et morcelées" (p. 41). Les essais consacrés au Quatrième siècle montrent dans quelle mesure le questionnement sur l'Histoire et sa mise en discussion constituent un premier noyau de la recherche glissantienne. Le peuple antillais qui part à la recherche de son histoire accomplit un voyage à rebours dans le temps, dans l'oubli et dans la souffrance, un voyage douloureux que l'imagination, la mémoire et le récit rendent possible. C'est le vieux quimboiseur, Papa Longoué, sorcier mais également gardien de la mémoire collective du peuple des dominés, des esclaves et de leurs descendants qui incarne cette pratique du récit. Elena Pessini analyse la présence et les fonctions de cette figure qui accomplit, à travers les histoires qu'il raconte, un exercice de relation, de prise de conscience, de dialogue avec l'autre. À travers la voix du quimboiseur, la parole remplace le cri de douleur et le silence originels qui ont présidé à la naissance des esclaves. Le personnage de Mathieu Béluse, héritier moderne de la parole du quimboiseur, devient écrivain et alter ego de Glissant dans Tout-monde et dans Le traité $d u$ Tout-monde. Nous passons de la deuxième à la troisième partie de l'ouvrage qui montre comment les romans de l'écrivain martiniquais inaugurent une nouvelle phase. Dans une pratique d'écriture qui mêle de plus en plus le roman et l'essai, Glissant se fait l'interprète d' "une vision nouvelle qui sans le forcer accepte le désordre du monde" (p. 97). Carminella Biondi et Elena Pessini montrent dans quelle mesure le lecteur, et Mathieu, sont conduits dans une sorte de parcours initiatique vers la prise de 
conscience d'un monde fait de relations imprévisibles, imprédictibles pour le dire en langage glissantien, et pour cela même, cohérentes.

3 Les pages de présentation du volume annoncent aussi une autre évolution de l'œuvre théorique glissantienne qui semble avec le temps gagner en clarté, s'imposer par ses reprises, ses redites, ses approfondissements, qui exprime la volonté d'être comprise par le lecteur. La recherche de nouveaux termes et de nouvelles définitions n'introduit pas des thèmes nouveaux, mais approfondit, éclaire et complète les idées-clefs qui agissent comme des constantes. Au contraire, comme l'illustre Elena Pessini, dans le chapitre (inédit) qui clôt le livre consacré au tout dernier roman Ormerod (2003), la production romanesque semble défier le lecteur, le situe face à un matériau brut à déchiffrer, à des indices truqués et le contraint, parfois, à accepter humblement de ne pas tout comprendre. Dans Ormerod, par exemple, "l'idée même du point de vue, d'identité et de personnage joue sur des contours extrêmement flous [ ... ] le lecteur est à la fois invité à accepter le vertige, mais à être aussi capable d'y mettre un certain ordre pour que tout ne soit pas que tourbillon" (p. 129). Ormerod est, pour Pessini, un roman qui réunit tout l'univers glissantien, dans lequel prend corps une vision "archipélique" du monde, un monde composé d'une multitude de diversités qui forment un tout. Ce roman, suggère le critique, établit un nouveau pacte entre le lecteur et l'écrivain, représente un défi et, en même temps communique, tant grâce à sa forme qu'à son contenu, l'instabilité du réel, plaçant ceux qui s'aventurent entre ses lignes "sur les pistes d'une littérature de l'inconfort et de l'inquiétude" (p. 130). En guise de conclusion, Carminella Biondi et Elena Pessini cèdent la parole à Édouard Glissant; le lecteur pourra découvrir quatre textes inédits de l'auteur, des réflexions sur l'écriture et sur les Antilles en forme de fragment. Le volume offre donc un parcours de lecture qui éclaire une œuvre considérée difficile, peu accessible, où s'exprime toutefois une pensée fascinante qui interprète les contradictions du monde qui est le nôtre. 